\title{
Emission and conductive properties of polyaniline thin films
}

\author{
Yu.M. Yumaguzin†, T.R. Salikhov, R.U. Shayakhmetov \\ †yum-yulaj@ya.ru
}

Bashkortostan State University, 32, Z.Validi St. 450074 Ufa, Ruusia

Thin films of polyaniline were prepared by thermal vacuum deposition from the Knudsen effusion cell. The surface condition and thickness of the deposited films were monitored on the basis of analysis of AFM images obtained by NanoScan 3D. Conducting properties of the films were studied at different sputtering conditions. To increase the conductivity of polyaniline layers, the temperature conditions of deposition from the Knudsen cell were selected. The temperature range of $500-550 \mathrm{~K}$ proved to be the most optimal. In addition, protonation of the freshly prepared films in vapors of hydrochloric acid solution was carried out. For polyaniline films a conductivity value of $1.0 \mathrm{mS} / \mathrm{cm}$ was achieved as a result. The main part of present work investigates polyanyline thin films with thickness of $30 \mathrm{~nm}$ on the tungsten-spike cathodes by means of field-emission measurements. The emitted electron energy distribution as a function of the electric field was obtained. The current - voltage characteristics and coating structure were also studied with the use of a field-emission microscope. The results obtained were compared with those of a pure-tungsten cathode. It is experimentally shown increasing of emissivity of tungsten tip coated with a thin film of polyaniline. The effective temperature of emitted electrons from tip with a film of polyaniline was determined according to the analysis of the energy distribution. Evaluation of electron temperature leads to a value of about $2300 \mathrm{~K}$, which corresponds to $0.2 \mathrm{eV}$ on the energy scale. The paper considers the phenomenon of charge carriers transfer in the system of metal - polyaniline - vacuum and the model of the energy bands of metal - polymer film contact.

Keywords: polyaniline, Knudsen cell, electron emission.

In the recent years electrophysical properties of highmolecular poly-conjugated compounds - electroactive polymers are studied intensively. It has been shown that the representatives of this class - polyanilines (PANI), specifically thin film structures on their base, are promising for the development of nanoelectronic devices such as LEDs, solar cells and field transistors [1]. To provide optimum work of electronic devices the development of special technologies of polymer coatings with controlled thickness and specified morphology for different substances are relevant. This is a complicated objective as PANI, like other electrically conductive polymers, are classified as non-processable materials. The polymer is infusible and practically insoluble, therefore the traditional technologies of hot-melt or solution deposition are not applicable for it.

In this work the electric conductivity of thin films PANI obtained by thermal vacuum deposition is measured and emissive properties of these films applied on tungsten cathodes surface are studied.

For this purpose, a technology was developed to obtain thin films of polyanilines by the method of thermal vacuum deposition from a Knudsen effusion cell. The length of a cylinder cell of silica glass was $25 \mathrm{~mm}$, internal diameter $4 \mathrm{~mm}$, operating temperature varied within 500-650 K. Surface morphology and thickness of deposited films were controlled by an analysis of AFM images obtained by scanning nanohardness tester "Nanoscan 3D" (fig.1). Thickness of the studied films ranged within $30-300 \mathrm{~nm}$ (fig.1). All the obtained films were of the same thickness, their specific conductivity was about $1.0 \mu \mathrm{S} / \mathrm{cm}$.
To increase the conductivity of polyaniline layers the temperature regime of thermal vacuum deposition of the Knudsen cell was selected. The temperature range 500-550 $\mathrm{K}$ was proved to be optimum. Besides, as-prepared films were protonated in the vapours of hydrochloric acid solution [2]. As a result, the specific electroconductivity of PANI films amounted to $1.0 \mu \mathrm{S} / \mathrm{cm}$ and more. Experimental samples of solar cells and field transistors were obtained on the base of studied films.
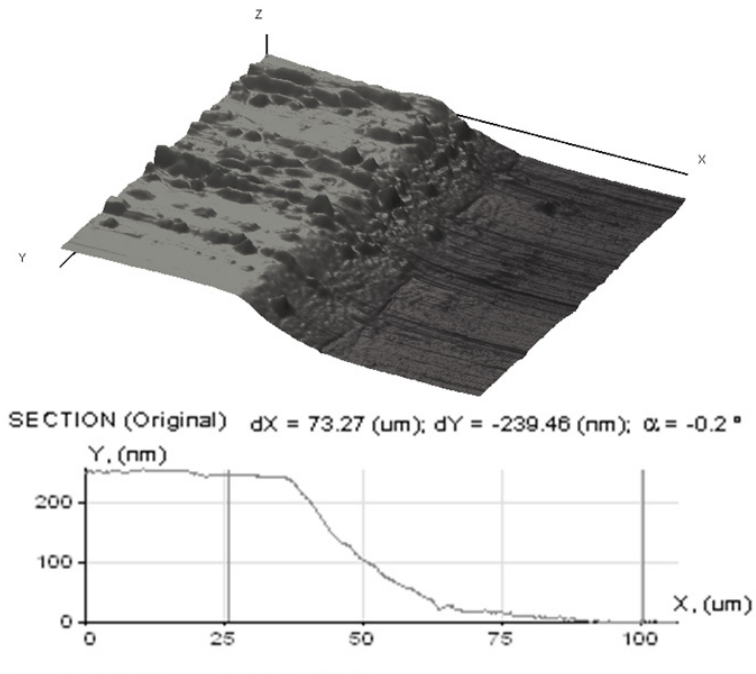

Length: $105.54 \mathrm{um}$; Height: $250.58 \mathrm{~nm}$

Fig. 1. Morphology of polyaniline surface on the base of analysis of AFM images and film edge profile. 
In addition, field emission of electrons from polymer (polyaniline) film deposited on tungsten surface by thermal vacuum deposition from a Knudsen cell in the conditions of ultra-high vacuum are studied in the present work. Voltagecurrent characteristics of tungsten with an initial (atomically clean) surface and deposited polymer film are measured.

Another aim of the work is to study energy spectrum of emitted electrons. Similar work was carried out earlier with another polymer, polydiphinilenphthalide. In these polymers one can achieve an anomalously high level of conductivity without doping [3-5]. High conductivity in these objects is observed if several conditions are fullfilled, the main of them being a small, less than a certain critical value, thickness of a polymer sample. Anomalously high level of conductivity in thin polymer films seems to be one of few consequences of nanoelectronic properties of the materials, i.e. electron properties specific to low-dimensional objects.

Studies carried out in the present work show a high emissive capacity of a cathode covered with polyaniline that can be seen in fig. 2. The studies were carried out on a facility including a field emission microscope and electron spectrometer. Energy distributions of emitted electrons were measured (fig. 3). As compared to a metal cathode without a deposition, the distribution is wider and displaced to $0.5 \mathrm{eV}$.

For making a zone diagram and interpreting data on voltage-current characteristics for the net current of the tip, use some average value for the work function of substrate, i.e. the emitting surface of tungsten tip and assume it to be equal to $\varphi=4.5 \mathrm{eV}$. Thickness $d$ of deposited film PANI is about 30 $\mathrm{nm}$. Let the band gap width $E_{g}$ of deposited material lies in an interval from a tenth to $3 \mathrm{eV}$ that corresponds to the range of the most frequent values of $E_{g}$ for polyaniline. Let's assume that film is deposited on an atomically clean and smooth surface of tungsten tip, i.e. taking into account the method of deposition, one gets an ideal contact metal/semiconductor without any air gap and chemical blocking layer. The deposited layer is considered to be in thermodynamic equilibrium with a substrate.

When the film is an intrinsic semiconductor with a small value of affinity to electron $\chi$, in a wide range of values $E_{g}$ the following condition is valid:

$$
\frac{E_{g}}{2}+\chi<\varphi
$$

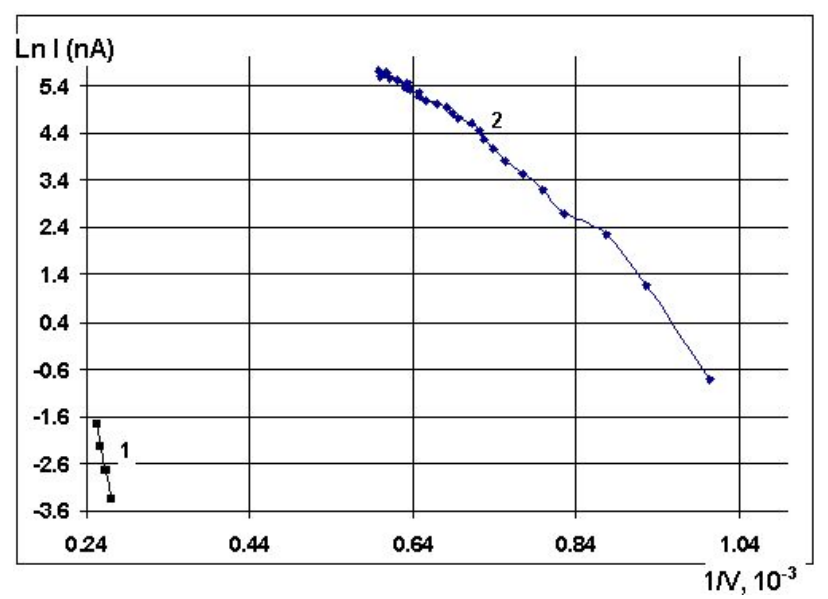

Fig. 2. Fowler-Nordheim characteristics for tungsten with initially clean surface (1) and deposited polyaniline film (2). and at that at the place of contact from the semiconductor side a depletion region, i.e. potential threshold for electrons injected from metal is formed.

Therefore, practically in any case of deposition on tungsten a depletion region of width $L$ is formed in the film. The bottom of conducting zone of semiconductor layer is always higher than the Fermi level of the system. As concentration of electrons in donor semiconductor polyaniline is comparatively small, simple calculations show that in this case that at the temperature of studies $(T=300 \mathrm{~K})$ $L>d$. Taking into account Schottky effect, this means a presence of a potential barrier but not a threshold, even if there is no external field. Probably, this is the reason of emission at considerably lower field in comparison with a pure tungsten tip.

One should point out that if the layers are thick, i.e. $L<d$, electron injection from metal Fermi level to the conducting zone of semiconductor film is not possible until the penetrating external emission field causes an additional inclination of energy zones in the film and changes potentional threshold into a barrier. From this, limitations on the maximum thickness of the layer on the tip becomes clear Namely, if the penetration depth of external field $\delta<d-L$, the injection of electrons into the conducting zone is not possible and it is necessary to attract another mechanisms to provide and explain stable supply of charge carriers to emitter surface: forming of conductive channels, conductivity in the energy zones of defects, grain boundaries, etc.

Penetration depth of external field can be determined from the following considerations. For metals $\delta=5 \cdot 10^{-9} \mathrm{~cm}$. As it is known, $\delta \sim n_{0}^{1 / 2}$. Then, for semiconductors we have $\delta=5 \cdot 10^{-9} \cdot\left(10^{22} / 10^{16}\right)^{1 / 2}=5 \cdot 10^{-6} \mathrm{~cm}$, i.e. about 500 atomic layers. Thus, in our case the field penetrates into the film completely. Note also that $\mathrm{d}$ depends on the applied voltage, but for thick layers this doesn't matter. Therefore, the thickness of deposited layer is considered to be optimum, if it meets the condition $\mathrm{d} \leq L+\delta$, where $\delta$ corresponds to a selected value of operation voltage. If this condition is fulfilled, a stable supply of charge carriers to the film surface, i.e. compensation of their removal from this region during emission is carried out due to their injection from metal substrate to the conductive zone of semiconductor film.

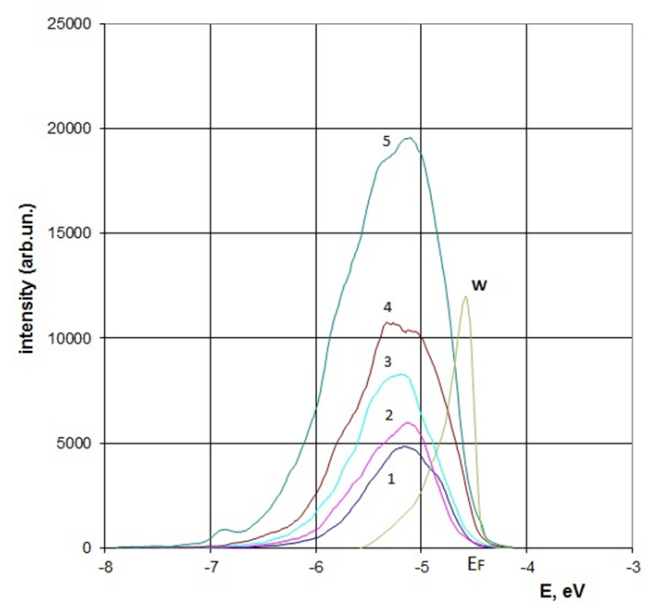

Fig. 3. Series of energy distribution of electrons on spike with polymer deposition at different emission tension $(1-1400 \mathrm{~V}$, 2-1420V, 3-1460V, 4-1500V, 5-1540V). 
The application of external field and its penetration into semiconducting film of small thickness causes inclination of energy zones along the whole layer. In our case of extremely small thicknesses and strong fields the situation is most probably realized when the bottom of conducting zone in subsurface region becomes lower than the system's Fermi level, i.e. a strongly degenerated region is formed. In the approximation of "zero current" the field value at which degeneration of electron gas in subsurface region of emitter occurs can be evaluated by the formula [6]:

$$
F \geq F_{0}=1.44 \cdot 10^{6} \varepsilon^{1 / 2}\left(\frac{m_{n}}{m}\right)^{3 / 4}\left(\frac{T}{300}\right)^{5 / 4}\left(\frac{\mathrm{V}}{\mathrm{cm}}\right),
$$

where $\varepsilon$ is the dielectric constant of semiconductor, $m_{n}$ is effective mass of electron, $T$ is absolute temperature in kelvins. For example, for silicon $F_{0}=5.1 \cdot 10^{6} \mathrm{~V} / \mathrm{cm}$, i.e. degeneration usually occurs at the field values that are considerably less of those required for field emission, therefore practically always emission goes from the degenerated conducting zone. Applied voltage is redistributed between the interval cathode/anode and decreases on a low conducting film. All the decrease can be considered to take place in the depletion region (due to a small layer thickness). It results in injection of carriers to conducting zone of the film.

Fig. 4 shows the form of energy zones in the case of auto-emission current in a strong field. The fall of potential $\Delta U$ occurs due to the current through the film, i.e. on the resistance $R$ of the layer. Therefore $\Delta U=i \times R$, where $i$ is the flowing current. The value of potential fall can be determined from a displacement of the energy distribution as compared to that for the pure metal surface of tungsten (fig. 4), which amounts to $\Delta E=0,5 \mathrm{eV}$. It should be noted that while measuring, Fermi level in the field electron spectrometer of a given construction always remains constant.

Injection current of electrons from metal into semiconductor is equal to saturation current $j_{s}$ in case of typical pass over the barrier and it can be calculated by diode theory of Bete [6]:

$$
j_{s}=\frac{1}{4} e n_{0} v_{T} \exp \left[-\frac{\Delta E}{k T}\right],
$$

where $v_{T}=\left(8 k T / \pi m_{0}\right)^{1 / 2}$ is average thermal velocity of electrons.

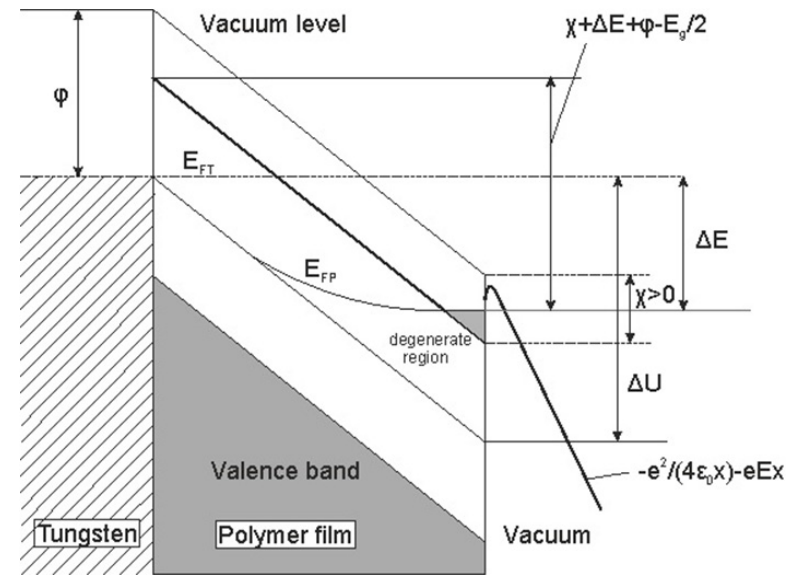

Fig. 4. Zone graph for the system tungsten spike/semiconductor film at strong external field application and auto-emission current.
It is known that average temperature of emitted electrons can be determined from the leading edge of energy distribution (fig.3) plotted in a semilogarithmic scale. Evaluation of electron temperature results in a value of the order of $2300 \mathrm{~K}$, which corresponds to $0,2 \mathrm{eV}$ in the energy scale.

Estimating the average thermal velocity of electrons, from formula (3) one can find concentration of electrons in the film for a known experimental value of saturation current density. According to the diffusion theory of rectification:

$$
j_{s}=e m_{0} \mu F_{0} \exp \left[-\frac{\Delta E}{k T}\right]
$$

where $F_{0}$ is intensity of electric field in semiconductor at the contact surface, $\mu$ is electron mobility. To take into account the mirror image forces both values are multiplied by the value $\exp (\Delta \varphi / k T)$, where $\Delta \varphi=\left(e^{3} F_{0} / \varepsilon\right)^{1 / 2}$ is a barrier decrease due to Schottky effect. Note that one uses formula (3), the value of saturation current is considerably less. We will consider the tunnel leakage from metal to conducting zone of the film and even directly to vacuum to be the main process.

In the case of thick films when injection by tunneling has a low probability, at temperatures meeting the condition $k T \geq \varphi-\chi$ (fig. 4), the classical pass over the barrier will be the main process of electron transfer through the contact zone.

From the analysis of energy distributions, effective temperature of emitted electrons from polyaniline film has been determined, a phenomenon of charge transfer in the system of metal-polyaniline-vacuum has been analyzed and a model of energy zones for metal-polymer film contacts has been developed.

Acknowledgements. The work was supported by the grant of RFBR 14-02-970008.

\section{References}

1. R.B Salikhov, Yu.N Biglova, Yu.M.Yumaguzin, T.R. Salikhov, M.S. Miftakhov, A.G.Mustafin. Techical Physics Letters. 39, 10, p. 854-857 (2013).

2. V.F. Ivanov, A.A. Nekrasov, O.L. Gribkova, A.A. Vannikov. Electrochimica Acta. 41, p. 1811-1814 (1996).

3. Yu.M. Yumaguzin, V.M. Kornilov, A.N. Lachinov. Journal of Experimental and Theoretical Physics. 103, 2, p. 264268 (2006).

4. R.M. Gadiev, A.N. Lachinov, V.M. Kornilov, R.B. Salikhov, R.G. Rahmeev, A.R. Yusupov. Pis'ma v JETF, 90, 11, p. 821-825 (2009) (in Russian) [Гадиев Р.М., Лачинов А.Н., Корнилов В.М., Салихов Р.Б., Рахмеев Р.Г., Юсупов А.Р. Письма в ЖЭТФ, т.90, вып.11, с. 821-825 (2009)].

5. R.B. Salikhov, A.N. Lachinov, R.G. Rakhmeyev. Molecular Crystals and Liquid Crystals. 467, 1, p.85-92 (2007).

6. R.Fisher, H.Newman. Avtoelectronaya emissia poluprovod-nikov. M. Nauka (1971) 216 p. (in Russian) [Р.Фишер, Х.Нойман. Автоэлектронная эмиссия полупроводников. (Пер. с немецкого под редакцией Г.Н.Фурсея). М. “Наука” (1971) 216 с.] 